\title{
Erratum to: What is the Accuracy of Nuclear Imaging in the Assessment of Periprosthetic Knee Infection? A Meta-analysis
}

\author{
Steven J. Verberne MD, Remko J. A. Sonnega MD, Olivier P. P. Temmerman MD, PhD, \\ Pieter G. Raijmakers MD, PhD
}

Published online: 3 April 2017

(C) The Association of Bone and Joint Surgeons( 2017

\section{Erratum to: Clin Orthop Relat Res DOI 10.1007/s11999-016-5218-0}

In the published study, "What is the Accuracy of Nuclear Imaging in the Assessment of Periprosthetic Knee Infection? A Meta-analysis" several errors can be found throughout the manuscript.

In Table 1, the column title, "Number of hip prostheses (total/infected)" should be: "Number of knee prostheses (total/infected)."
In Table 2, the column title, "Hip prostheses (primary/ revision)" should be: "Knee prostheses (primary/revision)." In the legend for Table 2, "H = hybrid hip prostheses" should be removed.

In the Discussion section, Figure 5A-B is incorrect as published. The corrected Fig. 5A-B is shown in this erratum.

The title of Table 14 is incorrect. The title should be: "Diagnostic accuracy of fluorodeoxyglucose-positron emission tomography for detection of periprosthetic knee infection."

The online version of the original article can be found under doi:10.1007/s11999-016-5218-0.

S. J. Verberne ( $₫)$, R. J. A. Sonnega, O. P. P. Temmerman Department of Orthopaedics, Noordwest Ziekenhuisgroe, Wilhelminalaan 12, 1815 JD Alkmaar, NWZ, The Netherlands e-mail: stevenverberne@hotmail.com

\section{S. J. Verberne, R. J. A. Sonnega, O. P. P. Temmerman} Centre for Orthopaedic Research Alkmaar (CORAL), Noordwest

Ziekenhuisgroep, Alkmaar, The Netherlands

P. G. Raijmakers

Department of Radiology \& Nuclear Medicine, VU University

Medical Centre, Amsterdam, The Netherlands 
Fig. 5A-B The pooled (A) sensitivity and (B) specificity of leukocyte scintigraphy in the assessment of periprosthetic knee infection with $95 \%$ CIs are shown

Fig. 8A-B The pooled (A) sensitivity and (B) specificity of FDG-PET in the assessment of periprosthetic knee infection with 95\% CIs areshown. FDGPET $=$ fluorodeoxyglucose-positron emission tomography.

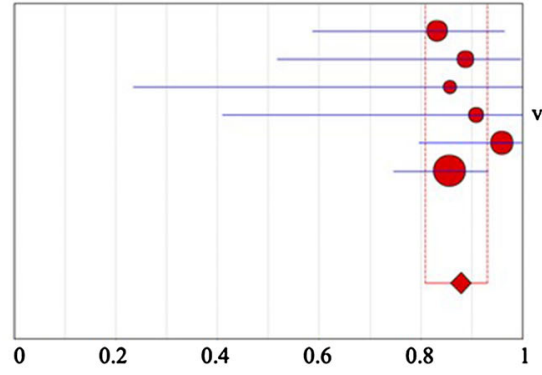

Rand et al. [38] Palestro et al. [34] Ooi et al. [28] van Acker et al. [50] Pelosi et al. [37] Kim et al. [18]

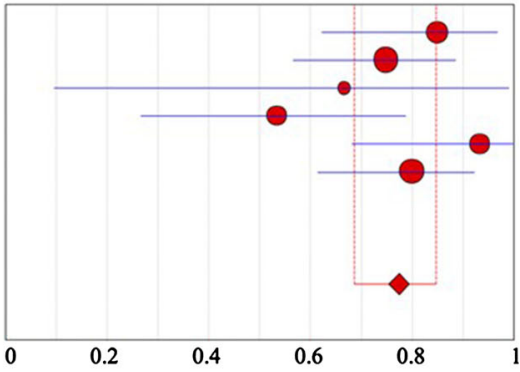

A

Sensitivity

Specificity

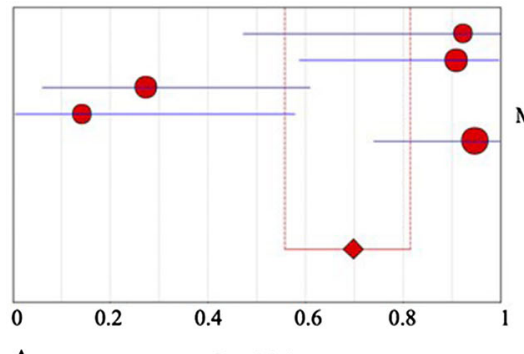

A Sensitivity

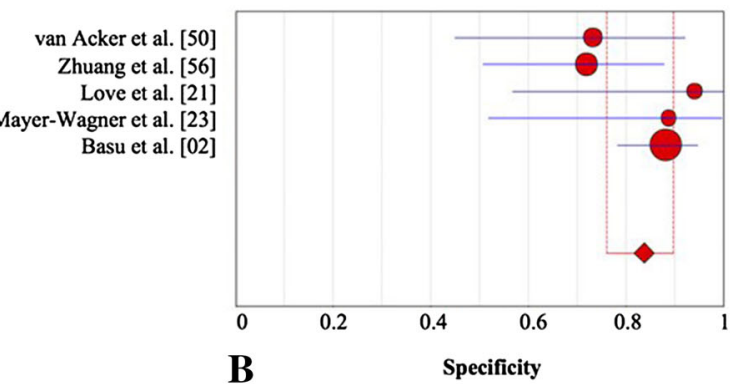

The title of Table 15 is incorrect. The title should be: "Study characteristics of fluorodeoxyglucose positron emission tomography for detection of periprosthetic knee infection."
Additionally, Figure $8 \mathrm{~A}-\mathrm{B}$ is incorrect as published. The corrected Fig. 8A-B is shown in this erratum.

The authors apologize for the errors. 